\title{
A Fieldwork of the Future with User Enactments
}

\author{
William Odom, John Zimmerman, Scott Davidoff, Jodi Forlizzi, Anind K. Dey, Min Kyung Lee \\ Human-Computer Interaction Institute \\ Carnegie Mellon University \\ \{wodom, johnz, scott.davidoff, forlizzi, anind, mklee \}@cs.cmu.edu
}

\begin{abstract}
Designing radically new technology systems that people will want to use is complex. Design teams must draw on knowledge related to people's current values and desires to envision a preferred yet plausible future. However, the introduction of new technology can shape people's values and practices, and what-we-know-now about them does not always translate to an effective guess of what the future could, or should, be. New products and systems typically exist outside of current understandings of technology and use paradigms; they often have few interaction and social conventions to guide the design process, making efforts to pursue them complex and risky. User Enactments (UEs) have been developed as a design approach that aids design teams in more successfully investigate radical alterations to technologies' roles, forms, and behaviors in uncharted design spaces. In this paper, we reflect on our repeated use of UE over the past five years to unpack lessons learned and further specify how and when to use it. We conclude with a reflection on how UE can function as a boundary object and implications for future work.
\end{abstract}

\section{Author Keywords}

User Enactments, Speed Dating, Design Methods, Prototyping, User Experience Design

\section{ACM Classification Keywords}

H5.m. Information interfaces and presentation (e.g., HCI): Miscellaneous.

\section{INTRODUCTION}

Designing radically new technology systems that people will want to use is difficult and complex. Design teams must draw on knowledge related to people's current values, desires, fears, and anxieties in order to envision a preferred yet plausible future. People's values and practices evolve with the introduction of new technology, and what-weknow-now about them does not always translate to an effective guess of what the future could - or should-be. New products and systems typically exist outside of current understandings of technology and use paradigms; they often have few interaction and social conventions to guide the design process, making efforts to pursue them complex and

Permission to make digital or hard copies of all or part of this work for personal or classroom use is granted without fee provided that copies are not made or distributed for profit or commercial advantage and that copies bear this notice and the full citation on the first page. To copy otherwise, or republish, to post on servers or to redistribute to lists, requires prior specific permission and/or a fee.

DIS 2012, June 11-15, 2012, Newcastle, UK.

Copyright 2012 978-1-4503-1210-3/12/06...\$10.00 risky. How can design teams more successfully investigate radical alterations to technologies' roles, forms, and behaviors in uncharted design spaces?

User Enactments (UEs) have been developed as a design approach that aids design teams in making this conceptual leap through the form of multiple enactments - what we call fieldwork of the future. Five years after their introduction [8], we reflect on our repeated use of this design method. In user enactments, designers construct both the physical form and the social context of simulated futures, and ask users to enact loosely scripted scenarios involving situations they are familiar with as well as novel technical interventions designed to address these situations. UEs emphasize bringing several visions of the future forward together to engage participants in critical exploration of what a preferred future state should be like. As participants enact the scenarios, the simulated social context activates, arouses, and challenges their social identity. UEs allow teams to observe and probe participants, grounding speculations about how current human values might extend into the future.

This perspective can help reduce risks that come with designing based on speculation, helping designers discover and focus on richer opportunities for technology to produce value. However, the extraction of these insights requires careful attention and many cycles of iteration. In this paper, we draw on several case examples of putting UE into practice and describe lessons learned. We reflect on how to use it, where it might be most beneficial, and how it impacts multidisciplinary teams that use it.

This paper offers two contributions. First, it discusses how and when to use UEs, unpacking the rich perspective it can provide about a particular target audience. Second, it proposes how UEs can function as a boundary object [28], creating a shared representation for participants and design teams; and creating a shared understanding and space for collaboration within multidisciplinary teams of, for example, designers, behavioral scientists and engineers.

In what follows, we first provide an overview of the UE method in terms of the general process as well as UE's relation to existing $\mathrm{HCI}$ design methods. We then describe and draw on case examples of three UE studies to unpack lessons learned and to further specify how and when to use the method. We conclude with a discussion of the method and reflect on how UE can function as a boundary object as well as implications for future research. 


\section{BACKGROUND}

UEs were originally described as part of a design method called Speed Dating [8]. Speed dating with UEs brings the metaphor of real-life speed dating for relationships into experience design. Real-life speed daters give up the focus provided by an outing with a single individual, for a series of timed encounters with multiple individuals. Speed daters usually learn relatively little about any single person they meet. However, their time with multiple partners can help them develop a wider perspective on the entirety of possible partners, and insights on what they really desire in a partner. User enactments, like speed dating in the real world, allows people to sample many alternative futures in the form of embodied, short enactments with design concepts that sketch out what kind of future might be. These futures are not always desirable; they can also be provocative, probing into edgy or unacceptable situations to expose possible underlying social boundaries. In this paper, we reflect on the application of UEs in several settings over several years, and describe the evolution of our understanding.

\section{The UE Design Process}

The design team begins by conducting in-depth review sessions of any collected field data and related literature. During these sessions, affinity diagrams and conceptual models are generated to create a shared understanding of the current reality and the design opportunities. The team then engages in several rounds of concept generation using methods such as brainstorming and bodystorming [2]. During this process, the team reflects on how the design ideas generated a map of the identified design needs and if the tacit knowledge accessed in the generation process seems to hint at aspects of the design space that might be more addressable than others. In other words, a core concern is whether the ideas proposed keep addressing the same issue, or continually address many issues from the same direction. This portion of the process generally results in approximately 100 design concepts.

The team then clusters concepts into thematic groups to understand the overall design space as well as more clearly articulate visions of preferred and undesirable futures. Next, clusters are iteratively filtered based on their fit to three areas: (i) the recognized needs and design opportunities, (ii) the importance of an underlying issue(s) a concept seems to probe, and (iii) the feasibility of realizing the concept through a user enactment. In the filtering process, several ideas are often merged together, resulting in a set of roughly ten design ideas.

These ideas are investigated by both rendering them in detailed, prose-based scenarios and investigating them via body storming to expose more nuanced social issues and aspects the design team aimed to investigate. These ten concepts are critiqued and filtered in terms of their fit to findings of prior work, importance of the probe, and ability to realize. This process can be seen in parallel to Schön's notion of design as a reflective conversation with materials [27]. In this case, the design material is narrative and the scenarios and bodystorming support this reflective conservation and help the design process move forward. Through repeated critique sessions, the UEs are narrowed down to a final set of scenarios.

At this stage, the team increases the fidelity of these scenarios by adding props and iteratively acting the scenes out until a consistent narrative flow is developed in and across all of them. This stage usually involves prototyping an environment (or "scene") for the scenarios to unfold in, as well as materials for each scenario. However, as we will describe later, the fidelity of the environment and concepts can vary significantly depending on the core questions being explored. Finally, many rounds of piloting are conducted to work out a detailed script that describes the actions of the researchers. The main focus of the piloting is to find harmony in terms of a participant's control. The user enactments need to not feel like complete exposition where the participant has no space to act, but they also must not be so free that the participant can wander away from the underlying design concept.

\section{Evolving UE: From Comprehensive Structure to Emergence and Iteration}

The original UE description focused on scenario development around a matrix. The design team first identified norms that shape today's behaviors, which might not clearly map onto the future. To examine how these contextual "risk factors" might affect user adoption of the technology, the team constructed dramatic scenarios, placing users into various social settings that reflect the cross product of these various hypothetical risk factors [see 8, p. 435]. This approach assumes design teams can accurately presuppose what the most critical issues will be. Practice with (and observation of) UEs, however, repeatedly identified that important issues more fluidly emerged through reflection across the many UEs teams and users experienced. Therefore, we abandoned the use of a matrix after our first use of UE.

The evolved UE approach benefits less from the structure of the scenarios than their number and the variety of futures that they enable users to experience. They allow designers to interactively adapt scenarios, vary applications, and alter the social and even physical context. Iterative refinement also allows the design team to engage users in ways that help probe on their values across a broad space of possible futures, and to verify the teams' assumptions about the future, pruning less realistic scenarios. Teams specifically design UEs that they think go too far, that cross social boundaries to confirm that they are there, or to discover that the teams' understanding of the situation is incomplete. This allows the design team to have a more grounded perspective of how various futures could be, and close in on which future should be. Similar to previous works that propose new design methods by reflecting on their 
application in several settings over several years [e.g., 12, $13,16]$, in the remainder of the paper we will describe how our understanding of UE has further evolved and lessons learned from the process. In what immediately follows, we first describe UE in the context of existing design-oriented methods in HCI.

\section{Related Work}

UEs build on several existing HCI design methods, such as scenario-based design [5] and experience prototyping [2], as well as the range of methods that embrace role-playing and performance as a critical means to engage users in exploration of potential technological futures [e.g., 3, 10, $14,17,20,21,23,30]$. Similar to the aim and ambition of many of these methods, UEs aim to tap into users' felt experiences of the present in order to provide insights into what the future should be. Like other existing methods such as Wizard-of-Oz [e.g., 15] and video sketches [e.g., 22, 31], UE aims to understand technology in an uncertain future.

At the same time, there has been a growing interest in how to support designers in considering user values throughout the design process. The Scandinavian tradition of participatory design has long been invested in engaging potential users in the design of new systems and technologies in the service of supporting their practices, desires and values [e.g., 18, 19]. Outside of this tradition, several other frameworks, such as value-sensitive design [11] and worth-centered design [6] have argued that designing technologies reflective of users' values can productively open the space for people to construct a deeper sense of value with these things. UEs share some of the same ambitions and interests; it aims to surface where value tensions may exist around future technologies and embrace them in moving from ideation to iteration. However, while approaches such as participatory design are clearly valuable and important, they may not be best suited for exploring complex, emerging design spaces where users may struggle to conceptualize new concepts. UE differs by requiring design teams to first create concepts embedded in scenarios, and then asking users to enact them to explore tensions and opportunities around potential near future technologies.

Finally, UEs differs from methods that tend to focus on one concept, such as experience prototyping [2] or technology probes [16], and emphasizes bringing several visions of the future forward to develop a better overall understanding of a design space. This approach is parallel to ongoing work describing the benefits of variety in ideation [e.g., 4, 9, 29].

\section{LESSONS LEARNED FROM THE UE PROCESS}

In this section we detail key lessons learned from doing UEs and describe how these issues can vary depending on the kinds of questions a design team aims to explore. We will focus on the following key issues. Control-balancing participants' relative level of control in the UE is crucial to generating worthwhile feedback. Fidelity-finding the appropriate level of fidelity for the scene is highly contingent on the nature of the questions the team aims to investigate. Ideation and Talkback-UEs enable design teams to rapidly test and iterate on the form of a design concept while retaining the same core goal. Intentional Provocation - including UEs constructed around clearly controversial or unacceptable technologies can generate rich insights about a target audience. Attention and Subtlety - that by their very nature UEs must to some extent direct participants' attention can complicate investigating ambient or peripheral technology scenarios.

Since its inception, we have applied UE in several different contexts. In this paper we describe and reflect on three specific cases, which present a variety in terms of participants, scope of questions investigated and overall UE fidelity. For each study, we describe a selected set of UEs we used, which are most pertinent to the lessons learned.

Study 1: Smart Home and Parenting. Our field research on dual-income families [7] indicated that parents want help in managing the busyness that comes with children's enrichment activities; things like soccer, ballet, piano lessons, etc. Our use of speed dating using needs validation (a type of speed dating done with storyboards) also showed that parents did not want a smart home that took over the role of parents; a home they felt might be raising children. Therefore, we designed UEs to help investigate what the appropriate role of a smart home might be. Specifically, we wanted to probe on issues of autonomy and control, planning and execution of events, improvisation when plans breakdown, and the home's role in observing and validating parental actions. This first UE project engaged 18 dualincome parents.

We constructed a low-fidelity smart home in a conference room. We used large sheets of white foamcore to create walls and the illusion of separate rooms. We drew home appliances, such as a cloths dryer and refrigerator on a floor to ceiling white board to give these products a place within the simulated home. Finally, we added small hand props such as a laundry basket and full shopping bags to help set the stage and give participants materials to handle during the encounters. Research team members played the role of the smart home, speaking or making affirming noises for the home and updating simulated information screens by adding and removing sticky notes.

UE 1: Arranging a Carpool. This scenario involves one participant in the role of parent, two absent children, and an absent spouse. We set the scene by telling the participant that soon they would need to leave home to pickup a child from an activity. We also informed them that their spouse was to pickup the other child. To make the scene feel more real, we used the names of the participant's children and the activities their children participate in. We then instructed the participant to begin preparing dinner for the family. As they play act cooking, a research member calls the participant on their real mobile phone. Playing the role of spouse, the researcher says they must work late and cannot make the pickup. The researcher then quickly hangs up 

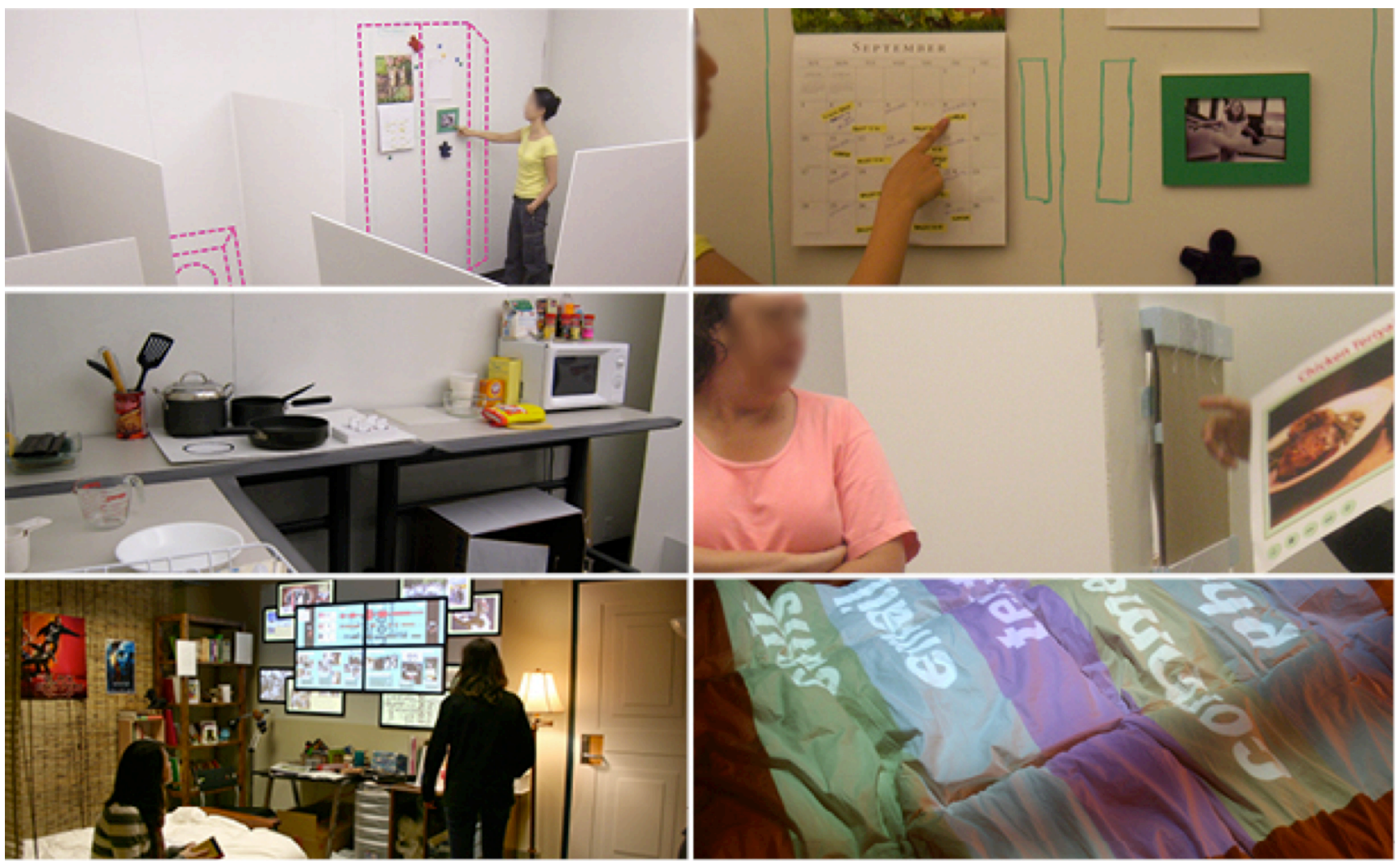

Figure 1. Top Left: The low fidelity smart home scene used in Study 1. Top Right: Information screen updates simulated with sticky notes for this smart calendar in Study 1. Middle Left: The slightly higher fidelity kitchen used in Study 2. Middle Right: Participant examining the meal planner application as a researcher updates the screen (Study 2, UE 2). Bottom Left: Screens redecorate as a friend enters the Teen Bedroom (Study 3, UE 1). Bottom Right: The Status Quilt that continually produced tepid reactions (Study 3, UE 2).

before the participant can negotiate. The participant now faces a dilemma; they cannot pickup both children before the children's activities end. The smart home has observed the interaction and it offers to help. For some participants, the house has proactively called neighbors to arrange a pickup. For others, the home simply communicates which neighbors it thinks might be available to make the pickup.

This UE probes on how proactive a smart home should be in taking on parenting tasks. It primes parents with the familiar situation of having carefully laid plans suddenly breakdown, and it builds on parents' underlying anxiety that they will leave their children unattended. Parents did not like the smart home helping with this task. Asking neighbors and friends raises complex issues of obligation parents felt a home could never handle.

UE2: Home Provided Validation. This UE begins at the end of a long day. The participant is in the kitchen and told that they have just completed all of their parenting tasks for the day. The smart home recognizes the completion of tasks and proactively engages the participant. The home compliments them on working so hard as a parent. As a reward, the home says they deserve a glass of wine.

This UE was intentionally crafted to probe how parents would respond to a system socially validating their parenting. We expected participants would view the home as crossing a boundary into socially inappropriate behavior.
We suspected they would view a computer that validates parenting as inauthentic. Interestingly, most participants appreciated the home's gesture. Their unexpected reaction hinted at how unappreciated they felt.

UE 3: Packing Reminder. During our fieldwork we observed that many parents placed all items needed for a child's enrichment activity into a bag, staged by the front or kitchen door. For example, the evening prior to a soccer practice, a parent would place the child's soccer bag by the door in preparation for the next day. Nevertheless, we also witnessed this coping strategy breakdown, as many families arrived at events only to discover a critical item was missing. This UE begins with the participant walking into the home carrying shopping bags. As they walk past the clothes dryer, a researcher playing the role of the smart home informs them that their child's soccer uniform is clean and in the dryer. The smart home is implying that now would be a good time to take this item and place it into the child's activity bag.

This UE probed the value of reminders situated in time and place, and of a reminder system that has a systemic view of all of the work and dependencies that goes into preparing for a child's enrichment activity. Interestingly, when running this UE, almost every participant shared that the home should tell their child the uniform was ready, even though children rarely participated in preparing for these 
activity bags. Parents recognized the possibility that technology could scaffold the work, allowing them to pass appropriate responsibilities to their children and to shift the focus from getting all of the work done to teaching their children responsibility. This was an entirely new idea for us on what smart home technology might do.

Study 2: Family Reminders. This UE also looked at the needs of busy dual-income families, but with a focus on reminders. From our previous fieldwork $[7,8]$ we observed that reminders were often deeply embedded in the performance of everyday routines, and that when families deviated from their routines, they often failed to undertake many tasks because they lost these embedded reminders. For example, a dad would make lunches for the kids while doing the dinner dishes. But if the family went out to dinner, there was a good chance the lunches would not get made. In addition, our fieldwork and prior speed dating sessions gave us the hunch that people enjoyed being reminded of the things they like to do; activities that help define them as a family. This second UE study engaged 12 dual-income families including both parents and at least two teenage children.

Once again we constructed a low-fidelity smarthome in a conference room with foamcore walls. This home focused mainly on the kitchen. It included a kitchen counter made from a long narrow table and covered with cooking tools and prop food, a dining table and four chairs, and the front door of a large refrigerator modeled out of foamcore. The fridge door had a screen where a research assistant would update paper interface screens in response to actions participants took.

UE 1: Family Conversation. This UE had the intention of stimulating conversation between teen and parents. One parent and the children were asked to work together to prepare dinner. The smart home then intercedes in the activity by teaching French to the parent and children as they work together. The home focuses on the foods and food prep activities to introduce new vocabulary.

The first time we ran this enactment it was a complete failure. The parent viewed this activity as educational and took it very seriously. The children had a very negative reaction when their parent assumed a teaching role, and not soon after the start, the simulated smart home had managed to completely end all communication between the parent and children.

Reflecting on this first session, the research team generated a new UE with the same intention of conversation. This time they situated it as a trivia game to be played in the car, a place parents and children often spent a lot of time together. The trivia game included questions about books and movies the family had read or seen together as well as trivia based on family holidays and special occasions. In addition, the game was rigged to increase the chances of the children beating the parents.
This UE probed the role of a smart home in creating the atmosphere for people to "do family." It was literally a reminder for them to talk to each other and stay connected. This second version of the enactment proved much more successful in generating conversation and interaction than the first design. It also reinforced that when parents play games with children, children enjoy winning and parents enjoy seeing the whole family being happy together.

UE 2: Meal Planner. This UE began with the dad and the children in the kitchen. The kids were doing homework and the dad was preparing to go to a meeting. The mom was expected home soon, to make dinner so that the dad could go to a meeting. As the scenario begins, the mom comes home and informs the dad that she must work late and that he needs to make dinner. The dad turns to the screen on the refrigerator; an interface to a smart cooking system. The fridge makes several recommendations based on the current inventory, the perishability of different foods, and the preferences of family members. The system encourages the family members present to work together to select what they would like from the limited set of choices.

This UE probed the challenges of improvising, especially when improvising a task where the other parent has most of the domain knowledge. In this case we had observed that many moms knew the inventory of the kitchens and many dads did not.

UE 3: What We Like To Do. We began this UE by asking a parent to clean the house. While they cleaned the kitchen, several photo frames around the kitchen updated their images. They displayed pictures from a family holiday and pictures of one of the children dancing ballet. Prior to the enactment, the parent had been primed with information that the family would soon go on vacation, and that the main enrichment activity for this day was ballet lessons.

The UE was intended to function as an ambient display, reminding the parent of what they liked to do and of who they were as a family. This was intended to be a counterpoint to most reminder systems that focus on showing people all the work they still need to do. Interestingly, most participants focused on the cleaning task and took little notice of the changing frames.

Study 3: Virtual Possessions in a Teen Bedroom. Our field research [25] revealed different ways teens worked to make their virtual possessions (e.g., photos, music, social networking information, text messages, etc.) more present in their bedrooms and how they drew on them as resources for self-reflection and self-presentation to different social groups. This UE project [26] engaged 14 teenagers to probe how increasing the presence of a teen's virtual things in their bedroom through radically new forms and behaviors would shape perceptions of their value. For this study we created a higher fidelity scene. We constructed the room in our lab with used-furniture and scattered popular culture objects (e.g. posters, books, music) and common items (e.g. 
clothes, school papers). A major addition to the room included twelve overlapping displays made from black and white foamcore that fill the wall above the teen's desk. We used an HD projector to create the illusion that they functioned as independent screens. We created a set of displays that could be easily integrated into the bedroom, while at the same time might be perceived as overwhelming. We hoped this tension might provoke teens to reflect on the amplified presence of technology in their personal space.

UE 1: Redecorating Bedroom. The participant enters the bedroom to prep for an upcoming exam and finds their displays showing various things, including: a visualization of new digital messages, photos of a wild party, provocative pop-culture images, etc. The teen receives a text message indicating a friend is coming over to study much earlier than anticipated. The friend (played by a confederate) soon enters the room, triggering five of the screens to automatically "redecorate". The screens highlight events both attended, images from parties, digital communication patterns, and images of the two friends in Halloween costumes from a time before they knew each other.

The UE probed on whether ceding autonomy to a system to present information about teens to other people would be perceived as beneficial or as complicating teen's sense of authentic self-presentation. We also wanted to probe how far back "too far back" is for teens as the system surfaced images of the friend from before they knew each other. Teens overwhelming reacted against the auto-redecoration. It was perceived to evoke inauthentic presentations of self and be a distraction from the face-to-face work of building a friendship. Surprisingly, teens largely did not react against the system surfacing information about their past; in several cases they desired to go even farther back to explore what their lives were like previously and how they have changed.

UE 2: Virtual Possession Status Quilt: The participant enters their room at night to prepare to go to sleep. On their bed they find an illuminated quilt displaying information about recently updated, unread virtual things (e.g., new Facebook wall posts, photos and comments they are tagged in, new texts and emails). The quilt indicates they have no unchecked items. After taking note of this, they turn the light off, take off their shoes, and climb into bed, settling under the quilt. While they pretend sleep with their eyes closed, the data on the quilt changes, indicating they have new content waiting for them. After several moments, a confederate playing a parent frantically knocks on the bedroom door, stating the school bus will be arriving soon. They get up in a rush, taking note of the changes on the quilt before hurrying out of the door.

We used the status quilt to probe on how teens would react to the significantly amplified presence of their changing virtual possessions. We wanted teens to confront what it feels like to be surrounded by - and wake up in-their virtual things, and how this might shape their existing bedroom practices and routines. This UE produced tepid reactions from teens. They typically went through the motions of the scenario, but were largely ambivalent about their experience and, when prompted, rarely found the quilt to be subtle or peripheral.

UE 3: Multiple Selves. Sitting in their bedroom after school, the participant views four different versions of their social networking profile targeted at four different audiences: family, school friends, sports team, and church. The teen is able to post status updates, comments and other content to each group exclusively. After a few moments they receive a text message on their phone from a sports team friend, causing a highlight in friend quadrant of the 12-screen display. A few moments later a different quadrant indicates another text message has arrived from a school friend. The UE concludes when a parent asks them to get ready for sports practice.

This UE probes whether teens see themselves as whole or as a collection of social roles. We wanted to explore if they felt discomfort by being confronted with a fragmented sense of self. We grounded the scenario in incoming communication to illustrate its link to social audiences external to the bedroom. Surprisingly, teens appeared quite comfortable when faced with seeing their self as multiple, curated selves. They felt the fragmentation could help make their lives more manageable as they move between different social spaces, both virtual and real, in their everyday lives.

In the next section, we draw on examples from these studies to describe key lessons learned through repeated using UE.

\section{Balancing participants' level of control}

Creating scenarios that enable participants to have an experience they can relate to is directly implicated in evoking behaviors and feedback useful to investigating the UEs' core questions. Effectively directing participants and delicately balancing their relative control to act freely within scenarios is crucial to achieving this goal.

At the beginning of each UE researchers provide participants with a brief background on the course of events leading to the scenario and, in most cases, what their task will be in it. The level of detail provided in this briefing requires careful attention. Too little detail can leave participants confused, and lead to unanticipated breakdowns during the enactment. Too much detail can have an overwhelming effect, causing participants to become hyper aware of their behaviors. Both instances can cause participants to look to outside of the scene in attempts to clarify what they perceive researchers "want" them to do. The goal is to make participants comfortable enough to draw on their own experiences to engage with and make sense of the enactment.

For example, the initial structure of Study 3, UE1 (Redecorating Bedroom) consisted of the teen entering their room to study for an upcoming exam. They soon receive a text message indicating a friend will be coming over earlier 
than expected - a choice intended to cause tension in the UE by amplifying the stress of studying. The teen is expected to rush to prepare for the exam; when the friend arrives the focus of the enactment should shift to the set of auto-redecorating displays. However, piloting revealed that we had not provided enough detail to guide participants. Initially, we brought participants into the bedroom, briefly introduced them to "their" material possessions and digital content on the screens in the room. During this briefing, we subtly alluded to possibility of the screens changing without providing any details about how, when or why. It was clear after several rounds of piloting that we had not provided enough structure. Participants often directly asked us what they "should" be doing and frequently missed cues indicating the screen content was changing. Post-enactment interviews often focused on participants seeking assurance over whether they had done the enactment "right."

However, we overcorrected when attempting to adjust for this lack of direction in our next iteration. We created a script that explicitly brought attention to the changing displays through structured conversation about each of them. The detail-laden script made the social interaction too forced and overly constrained the setting, leaving little room for the participant to guide portions of the enactment. Participants appeared preoccupied with looking for the next step and responding to the confederate with what they thought we wanted to hear, as opposed to drawing on their own experience to make sense of the unfolding situation.

This instance exemplifies the challenge we have faced across our applications of UE: finding harmony between giving participants too much freedom within the scenario on one hand, and making the scenario mostly exposition on the other. We have now incorporated the following changes to our practice to more effectively find an appropriate structuring for participants. First, begin the series of enactments with a scenario that has the goal of introducing participants to the familiar setting. This helps participants ease into their performative role, as opposed to requiring them to immediately make sense of a complex and potentially uncomfortable situation. For example, we introduced teens to the bedroom as "their room" and then asked them to reflect on their everyday routines, practices and behaviors before beginning the scenarios. This helped establish rapport with researchers and begin to prompt participants to engage with the UEs against the backdrop of their own experiences.

Second, focus on familiarity: ground participants in a familiar task to help them ease into the scenario. For example, we asked teens to study for an exam and then had the system's interruption of this task (coinciding with the friend arriving) help move attention to the core part of the scenario. Third, when using confederates to help probe particular aspects of a UE, craft their direction to loosely guide the flow of the scenario with planned points for participant engagement that are fluid enough to move on immediately if needed. For example, after the prior two rounds of piloting, we re-adjusted the confederate's role in the Redecorating Bedroom to subtly point out the screens as they changed, while leaving space to elicit reactions from participants (or to move on without forcing an answer). Further piloting revealed this was effective when the focus of post-enactment interviews shifted from participants questioning their performance to critiquing the design concept; they drew on stories from their own lives to support their rationales for why aspects of the UE supported and conflicted with their current values and practices.

\section{Finding the right level of fidelity for the UE}

The fidelity of the UE setting and narrative can vary considerably depending on the kinds of questions it aims to investigate. Finding the right level of fidelity centers on creating UEs evocative enough to get participants to "buy in" and channel their own experiences when enacting the scenario. At the same time, it is crucial to avoid over committing time and resources to unnecessary scene development. Study 2 and Study 3 provide exemplary cases of how the "right" level of UE fidelity can vary.

In Study 2, the fidelity of the domestic setting was low; the home walls were constructed with foam core boards and tape and the food was all made of plastic. These materials provided just enough context for participants to recognize the space as a kitchen (e.g., the kitchen versus laundry room). Low-fidelity worked for this set of UEs because of the high stress and arousal of the situation prior to the technical intervention. In UE 2 (Meal Planner), when the mom calls to say that she is not coming home and dad must prepare dinner, we witnessed the dads begin to sweat. Even with the low-fidelity surroundings, their experience of being in this situation and feeling stress in this situation was quite real and quite present. We could have constructed a more realistic environment, but it was not needed to connect participants with their experiential memory, because the memories were visceral and strong.

In contrast, Study 3 required a setting that reflected the intimate and personal nature of a teen's bedroom. To make the scene feel more familiar we paid close attention to detail in the overall aesthetic of bedroom as well as how technology was integrated into it. Our lab bedroom was intentionally crafted to appear 'messy' to reflect the composition of bedrooms we observed in the field and to help move away from the sterile, orderly setting of our lab. When we initially introduced teens to the bedroom, several participants (and their parents) commented on how it resembled the messiness of their own rooms. Additionally, we iterated on the 12 screen foam core displays several times to generate a form that "fit" into the design of the room. We wanted to explore tensions around technology in the bedroom, while avoiding having the sheer presence of the screens overshadow the content projected onto them.

Despite these efforts, early piloting revealed that something was still wrong. Our lab bedroom produced feelings of 
uneasiness and awkwardness for the participants - and for us. However, when we began to softly play music, selecting songs popular among US teenagers, this somehow worked to de-emphasize the lab and to make the bedroom feel like a real place. It may seem subtle, but this choice significantly helped participants feel more comfortable and to suspend belief. The music enabled teens' to subtly shift their attention away from the bedroom props toward enacting the scenario at hand.

These examples illustrate how finding the right level of UE fidelity is highly dependent on the kind of questions that ground the scenarios and, concomitantly, the kind of behavior that will be productive to investigating them. Importantly, Study 2 and Study 3 highlight how iterative cycles of piloting and rapid alteration to the UEs' form helped find the right levels of fidelity respectively without making an over commitment to extensive scene development.

\section{Ideation and Talkback}

UEs enable design teams to rapidly test and iterate on the form of a design concept while retaining the same core goal and questions. For example, if an enactment initially fails, the design team can rapidly develop a new concept to test. Importantly, this changes how UEs fit into the design process. UEs can be used to probe user reactions and gauge how on-target design teams are with evoking meaningful reactions and ultimately achieving their end goal. Beyond leading to new design spaces, UE can help design teams understand when going forward with an alternate design space would have been disastrous.

For example, UE 1 (Family Conversation) from Study 2, in which the smart home attempted to stimulate conversation between parents and teens by teaching French, was a complete failure. The design presented yet another opportunity for parents to be disappointed with their children. In redesigning this scenario for the following families we shifted from evaluating back to more generative design activities. The lightweight nature of the enactments supports this rapid transition between design actions. Working with the props at hand, the team rearranged the dining chairs to simulate being in a car. We were ready to try our new design one day after our initial failure. Our failed UE functioned as a boundary object to mediate discussions about why it was not working and, ultimately, to re-focus on a more appropriate context. The method allowed the team to fail quickly, to learn from the failure, and to rapidly push back with a new idea.

In other instances, such as Study 3 UE 1 (Redecorating Bedroom), we misjudged what we thought would be ageappropriate popular culture content for teenagers, which was projected on the 12 -screen display. Initial piloting revealed that teens often became distracted by this content, which derailed the enactment. It appeared this was not due to the content (e.g., musical groups, films) being in conflict with their own tastes, but rather that they perceived it to be more well matched for one generation younger than them. We were able to quickly adjust the form of the displays with new content, which resolved this issue in the piloting sessions that followed. Interestingly, while we have not directly utilized it, we imagine this virtue of UEs could be used in the inverse direction: seeing an unexpected behavior emerge and radically altering the form to bring this behavior out in the scenario.

Intentional Provocation through Controversial Concepts Intentionally situating UEs around design concepts likely to be seen as controversial, unacceptable, or infeasible can generate rich insights about a target audience, especially when participants do not have the anticipated negative reaction. For example, Study 3, UE 3 (Multiple Selves) explicitly presented teens as fragmented individuals. We used college students as stand-in participants when piloting this enactment. As we anticipated, they were disturbed by the idea that they would explicitly present a different view of themselves to different social groups. This made it so much more interesting to have the teens see such a value in this segmented display and to ask for one that could support six different selves. It reinforced for us the idea that teens who live in their parents' home and spend time under the often strict structures of high school social status simply have a very different view of the world. It also helped us understand how teens work to construct a self-identity as they move between their home, bedroom, school and other social spaces they have differing levels of control over.

In Study 1 UE2 (Home Validation), we aimed to investigate how parents would react to the home's recognition of their effort. Instead of seeing the situation as contrived or offensive, parents appreciated the sentiment; they seemed happy that someone, or in this case some thing, was noticing their hard work. In discussions with the parents they shared how they could do a chore correctly 100 times and it would never be noticed, but if they made one mistake, like packing the wrong child's water bottle in their school lunch, then this was the event the child always noticed and always remembered. We did not leave this enactment thinking technology should validate people, but we did have a greater sense for the felt life experience of being a parent.

What we want to draw attention to in these examples is how exposing participants to controversial concepts produced rich, unexpected insights about the lives of our target audiences. In the case of teens, it helped us better understand how they fluidly manage their presentations of self in and across real and virtual settings in complex and unique ways. Whereas in the case of parents, helped us see how experiencing a lack of validation can affect parents' everyday lives. Collectively, these insights helped guide our understanding of where opportunities might exist in these respective design spaces, even though we clearly never intended to pursue the actual design concepts. 


\section{Attention and Subtlety: Limitations of UE}

By their very nature UEs must to some extent direct participants' attention, which can severely complicate investigating ambient or peripheral technology scenarios. Ambient technologies are often too subtle for participants to notice in the context of a UE, and when they are directed to do so, the application becomes no longer ambient.

For example, Study 2 UE 3 (What We Like To Do) centered on several smart photo frames that would subtly change to remind parents of upcoming family events and enrichment activities their children would be going to that day. In the UE, we distracted parents with the task of cleaning the house. However, their attention rarely deviated from clearly for the UE's entire duration. In Study 3 UE 2 (Virtual Possession Status Quilt), we expected teens to react strongly against the presence of the quilt, particularly after "waking up" in the updated statuses of their virtual things. However, teens were typically unsure what they were supposed to take away from the scenario, and often quickly went through the motions to complete it. In both cases, having the scenarios grounded in an ambient technology that participants were not supposed to focus their full attention on at any key point made it difficult to have enough focus and structure necessary to run an effective enactment. Despite several rounds of iterative piloting and changes, we were unable to generate compelling experiences and eventually dropped both UEs from their respective studies.

\section{DISCUSSION AND REFLECTION}

UE provides a method for doing fieldwork of the future to help design teams take the conceptual leap into making new applications in uncharted design spaces when they have few social or interaction conventions to draw on. UE requires users to enact scenarios in which they get glimpses of several potential futures and to use their own experiences to critically make sense of what they encountered. This approach helps reduce risk, and it reveals new opportunities that would have been difficult to uncover in studies of people's current behaviors. However, UE can be a complicated process that requires careful attention to several factors through several iterative cycles of prototyping and piloting. A core contribution of this paper is to detail our experiences of repeatedly using UE in different cases to articulate when and how to use the method successfully. We have described how issues such as control, fidelity, ideation and talkback, intentional provocation, and attention and subtlety are crucial to consider throughout the UE process; however, they can manifest in different ways depending on the kinds of questions the design team aims to investigate. Here we describe additional benefits of UEs for participants, design teams, and the constituent disciplines of HCI.

For participants trying out the UEs, the method is critical in suspending belief to experience glimpses of a number of potential futures. Whether the context was a foam-core kitchen or a very realistic bedroom, we witnessed our participants experiencing the imagined situation in a realistic way. In addition, they were able to call upon their own prior experience to make sense of the scenarios. The UEs is also a unique evaluation method because they can explicitly provoke participants to experience controversial or negative concepts in ways that traditional prototypes and scenarios typically do not. This continues a tradition in design research of using fiction or pastiche scenarios as a resource in user centered design [e.g., 1, 24].

For the design team, UEs are instrumental in making the conceptual leap from data to concept, for covering a design space or making many iterations of one concept, and for exploring yet to be designed technology and services. In this way, UEs help the design team in reducing risk in what to build. They also function as boundary objects [28], allowing two or more distinct communities to interact and co-join around a mutually comprehended representation (or 'object'). In our context, UEs work as a boundary object by creating a shared reference to mediate ongoing dialogue between participants and the design team.

On a broader level, UE can work as a boundary object to connect constituent disciplines of HCI by providing a common context to mediate collaboration among multidisciplinary teams. For example, UE can help bridge the contributions of social scientists, which provide rich insights into people's current values and practices, and technologists, which develop new technical materials, but not necessarily with concrete ideas of how they will manifest in people's lives. In this way, UEs can link the competencies of these team members with designers to investigate new forms of technical applications and the potential social-technical futures they might inhabit. This can be critical to informing and reaching consensus on the next stages of development for multidisciplinary teams.

While we did not initially intend for the method to function in this way, it has and continues to. For example, findings from Study 1 and Study 2 eventually lead to three years of technical development of a smart calendaring system, and we are currently developing technology probes directly based on findings from Study 3.

\section{CONCLUSION}

In this paper we have described and reflected on our experiences of repeatedly using UE over the past five years and how it has evolved. We drew on three UE studies to unpack lessons learned across these cases and to further specify how and when to use the method successfully. In this, we aimed to emphasize how UE can help reduce risk in pursuing emerging design spaces and, importantly, the rich perspective it can provide on people's lives beyond studies of their current practices. Our reflection highlighted how UE can work as a boundary object to mediate dialogue between participants and a design team as well as among members of a multidisciplinary team itself. Ultimately, we hope this paper will help better support future research and 
practice aimed at critically investigating new and emerging uncharted design spaces, and the possible futures they might suggest.

\section{ACKNOWLEDGMENTS}

This work is partly supported by NSF grant IIS-1017429 and by Google. We thank the following people for their valuable help in developing User Enactments over the past five years: Bryan Crowe, Elena Kim, Ben Koh, K.C. Oh and Ray $\mathrm{Su}$ for the Smart Home and Parenting project; William Haines, Margeret Szeto, Yue Zhoa, Yong Woo Rhee, Joshua Ehlke, Xiang Shen, Zoe Ju Ouyang, Nina Shih, Sushmita Subramanian, Jing-Jing Tien, M. Louise Poythress, Dongwhan Kim, Phijarana Rattanathikun, Sanjay Arora, Hye-Eun You, and Daniel Weiss for the Family Reminders project; and, Hajin Choi, Stephanie Meier, Angela Park, and Alena Tesone for the Teen Bedroom project. We also thank the teen participants (and their parents), in addition to the 52 families who have taken over 250 hours from their busy lives to inform and inspire our research. Finally, we thank the myInfo team at Philips Research.

\section{REFERENCES}

1. Blythe, M., Wright, P. 2006. Pastiche scenarios: Fiction as a resource for user centered design. Interact. Comput. 18, 5, 1139-1164.

2. Buchenau, M., Fulton Suri, J. 2000. Experience Prototyping. Proc. of DIS '00, 424-433.

3. Burns, C., Dishman, E., Verplank, W., Lassiter, B. 1994. Actors, hairdos \& videotape-informance design. In Conference Companion of CHI '94, 119-120.

4. Buxton, B. 2007. Sketching User Experiences: Getting the Design Right and the Right Design. Morgan Kaufmann.

5. Carroll, J. (2000) Making use: Scenario-based design of Human-Computer Interaction. Cambridge: MIT Press.

6. Cockton, G. 2008. Designing worth-connecting preferred means to desired ends. Interactions, 15, 54-57.

7. Davidoff, S., Lee, M., Yui, C., Zimmerman, J., Dey, A. 2006. Principles of smart home control, Proc. of Ubicomp 06, 19-34.

8. Davidoff, S., Lee, M., Dey, A., Zimmerman, J. 2007. Rapidly exploring application design through speed dating. Proc. of UbiComp '07, 429-446.

9. Dow, S. P., Glassco, A., Kass, J., Schwarz, M., Schwartz, D. L., Klemmer, S. R. 2010. Parallel prototyping leads to better design results, more divergence, and increased selfefficacy. ACM TOCHI, 17, 4, Article 18

10. Ehn, P., Binder, T., Eriksen, M., et al. 2007. Opening the digital box for design work: supporting performative interactions, using inspirational materials and configuring of place. The disappearing computer, 50-76. Springer.

11. Friedman, B., Kahn, P., Borning, A. 2006. Value Sensitive Design and Information Systems. In Human-Computer Interaction in Management Information Systems: Foundations, 348-372.
12. Gaver, W., Dunne, T., Pacenti, E. 1999. Design: Cultural Probes. Interactions, 6, 1, 21-29.

13. Gaver, W., Boucher, A., Pennington, S., Walker, B. 2004. Cultural probes and the value of uncertainty. Interactions $11,5,53-56$.

14. Halloran, J., Hornecker, E., Fitzpatrick, G. et al. 2006. Unfolding understandings: Co-designing UbiComp in situ, over time, Proc. of DIS '06, 109-118.

15. Hartmann, B., Doorley, S., Kim, S., Vora, P. 2006. Wizard of $\mathrm{Oz}$ sketch animation for experience prototyping. Adjunct proceedings of Ubicomp 2006.

16. Hutchinson, H., et al. 2003. Technology Probes: inspiring design for and with families. Proc. of CHI '03, 17-24.

17. Iacucci, G., Kuutti, K., Ranta, M. 2000. On the move with a magic thing: role playing in concept design of mobile services and devices. Proc. of DIS '00, 193-202.

18. Iversen, O., Halskov, K., Leong, T. 2010. Rekindling values in participatory design. Proc. of PDC '10, 91-100.

19. Kensing, F., Blomberg, J. 1998. Participatory Design: Issues and Concerns. Computer Support Collaborative Work, 7, 167-185. Kluwer Press.

20. Kurvinen, E., Koskinen, I., Battarbee, K. 2008. Prototyping Social Interaction. Design Issues, 24, 46-57.

21. Laurel, B. 1991. Computers as Theater. Addison Wesley.

22. Mancini, C., Rogers, Y., Bandara, A., Coe, T., Jedrzejczyk, L., et al. 2010. Contravision: exploring users' reactions to futuristic technology. Proc. of CHI '10, 153-162.

23. Medler, B., Magerko, B. 2010. The implications of improvisational acting and role-playing on design methodologies. Proc. of CHI'10, 483-492.

24. Nathan, L., Friedman, B., et al. 2008. Envisioning systemic effects on persons and society throughout interactive system design. Proc. of DIS '08, 1-10.

25. Odom, W., Zimmerman, J., Forlizzi, J. 2011. Teenagers and Their Virtual Possessions: Design Opportunities and Issues. Proc. of CHI'11, 1491-1500.

26. Odom, W. Zimmerman, J., Forlizzi, J., Choi, H., Meier, S., Park, A. 2012, in press. Investigating the Presence, Form and Behavior of Virtual Possessions in the context of a Teen Bedroom. Proc. of CHI'12.

27. Schön, D., Bennet, J. 1996. Reflective Conversation with Materials. Bringing Design to Software, 171-189.

28. Star, S.L., Greisemer, J. R. 1989. The structure of illstructured solutions: Boundary objects and heterogenous problem solving. Distributed AI, 2, 37-54.

29. Tohidi, M., Buxton, W., Baecker, R., Sellen, A. 2006. Getting the Right Design and the Design Right: Testing Many is Better Than One. Proc of CHI'O6.

30. Wright, P., McCarthy, J. 2008. Empathy and HCI. Proc. of CHI '08, 637-646.

31. Zimmerman, J. 2005. Video Sketches: Exploring pervasive computing interaction designs. IEEE Computing, 4: 91-94. 